\title{
Hsp70 acetylation prevents caspase-dependent/independent apoptosis and autophagic cell death in cancer cells
}

\author{
YOO HOI PARK $^{1 *}$, JI HAE SEO ${ }^{2 *}$, JI-HYEON PARK ${ }^{*}$, HYE SHIN LEE ${ }^{1}$ and KYU-WON KIM ${ }^{1,3}$ \\ ${ }^{1}$ SNU-Harvard NeuroVascular Protection Research Center, College of Pharmacy and \\ Research Institute of Pharmaceutical Sciences, Seoul National University, Seoul 08826; \\ ${ }^{2}$ Department of Biochemistry, School of Medicine, Keimyung University, Daegu 42601; \\ ${ }^{3}$ Crop Biotechnology Institute, GreenBio Science and Technology, \\ Seoul National University, Pyeongchang 25354, Republic of Korea
}

Received April 12, 2017; Accepted May 22, 2017

DOI: $10.3892 /$ ijo.2017.4039

\begin{abstract}
Cancer cells are continuously challenged by adverse environmental factors including hypoxia, metabolite restriction, and immune reactions, and must adopt diverse strategies to survive. Heat shock protein (Hsp) 70 plays a central role in protection against stress-induced cell death by maintaining protein homeostasis and interfering with the process of programmed cell death. Recent findings have suggested that Hsp70 acetylation is a key regulatory modification required for its chaperone activity, but its relevance in the process of programmed cell death and the underlying mechanisms involved are not well understood. In this study, we sought to investigate mechanisms mediated by Hsp70 acetylation in relation to apoptotic and autophagic programmed cell death. Upon stress-induced apoptosis, Hsp70 acetylation inhibits apoptotic cell death, mediated by Hsp70 association with apoptotic protease-activating factor (Apaf)-1 and apoptosis-inducing factor (AIF), key modulators of caspase-dependent and -independent apoptotic pathways, respectively. Hsp70 acetylation also attenuated autophagic cell death associated with upregulation of autophagy-related genes and autophagosome induction. Collectively, these results suggest that the acetylation of Hsp70 plays key regulatory roles in cell death pathways as well as in its function as a chaperone, together enabling cellular protection in response to stress.
\end{abstract}

\section{Introduction}

Cancer cells are confronted with diverse environmental stresses including hypoxia, nutrient deprivation, and $\mathrm{pH}$

Correspondence to: Dr Kyu-Won Kim, SNU-Harvard NeuroVascular Protection Research Center, College of Pharmacy and Research Institute of Pharmaceutical Sciences, Seoul National University, Seoul 08826, Republic of Korea

E-mail: qwonkim@snu.ac.kr

*Contributed equally

Key words: Hsp70, acetylation, apoptosis, autophagy, ARD1, Apaf-1, apoptosis-inducing factor changes caused by metabolic byproducts and the tumour microenvironment (1-3). Stress factors induce diverse apoptotic signaling in cells, in which various pro-apoptotic proteins can be activated. To overcome the apoptotic response, cancer cells develop diverse ways to inhibit apoptotic signaling $(2,4,5)$. Furthermore, these signaling alterations can also allow cancer cells to resist chemo- or radiotherapeutic challenge (6).

The heat shock protein (HSP) 70 chaperone system is upregulated in many cancers and facilitates the refolding or degradation of proteins that are denatured as a result of stress (7-9). In addition, Hsp70 can also directly interfere with apoptosis pathways to protect cells (10-12). The diverse protective mechanisms of Hsp70 are known to confer resistance to some forms of stress-induced cell death.

Several pro-apoptotic proteins that are directly inhibited by Hsp70 have been reported. Apoptotic protease-activating factor 1 (Apaf-1), a key regulatory component of the caspasedependent apoptotic pathway, directly associates with Hsp70 (11,12). This interaction prevents oligomerization of Apaf-1 with procaspase-9, consequently inhibiting apoptosome formation. Another pro-apoptotic protein, apoptosis-inducing factor (AIF) is a mitochondrial intermembrane protein that initiates one of the key mechanisms of caspaseindependent apoptosis (13). Under stress conditions, AIF, which is normally well secluded in mitochondria, translocates to the cytosol and ultimately to the nucleus where it induces caspase-independent peripheral chromatin condensation and DNA fragmentation (14).

Autophagic cell death is another type of programmed cell death, which involves autophagy $(15,16)$. The crosstalk between autophagic and apoptotic cell death is a current topic of heightened interest. In relation to the HSP system, autophagy is a regulatory mechanism that maintains cellular protein homeostasis by sequestering and delivering large protein aggregates and whole damaged organelles to lysosomes for degradation (17). Previous studies have reported that autophagy is regulated by the heat shock response. Depletion of HSF-1 potentiates starvation-induced LC3 lipidation, which is associated with the formation of autophagosomal organelles, while Hsp70 overexpression inhibits this process (18). 
Recently, Hsp70 was reported to be acetylated by acetyltransferase arrest defective (ARD) 1 (Naa10, N $\alpha$-acetyltransferase 10) and this acetylation contributes to its protective function under cellular stress (19). Here, we sought to investigate the molecular mechanisms of Hsp70 acetylation-mediated cellular protection.

\section{Materials and methods}

Cell culture and stimulation. SH-SY5Y, HeLa and HEK293T cells were obtained from the American Type Culture Collection (ATCC). Cells were grown in DMEM supplemented with $10 \%$ fetal bovine serum and $1 \%$ penicillin/streptomycin in a $5 \% \mathrm{CO}_{2}$ humidified atmosphere at $37^{\circ} \mathrm{C}$. To induce cellular stress, cells were treated with $1 \mu \mathrm{g} / \mathrm{ml}$ doxorubicin or $0.3 \mathrm{mM}$ $\mathrm{H}_{2} \mathrm{O}_{2}$ for $24 \mathrm{~h}$.

Plasmid construction. Full-length cDNAs for human Hsp70 (Genbank: NM_005345.5) and human ARD1 (Genbank: NM_003491.3) were generated by PCR and subcloned into pCDNA3.1 (FLAG-ARD1) or pEGFP-C3 (GFP-Hsp70) vectors for cellular expression. For the construction of stable cell lines, cDNA constructs for Hsp70 and ARD1 were co-inserted into the pIRES vector, purchased from Clontech.

Transfection. Transfection was carried out as described previously (20). HEK293T cell transfection used polyethyleneimine (PEI) at a ratio of 4:1 (ml PEI/mg plasmid DAN) in basal media overnight, followed by a change of media. For the establishment of stable cells, pEGFP-C3-Hsp70 and pIRES-GFP-Hsp70-FLAG-ARD1 plasmids were transfected into SH-SY5Y cells using PolyFect reagent (Qiagen), according to the manufacturer's instructions. Transfected cells were maintained in complete DMEM with G418 $(500 \mu \mathrm{g} / \mathrm{ml})$. After several days, the surviving colonies were selected and amplified.

Antibodies. Anti-cleaved caspase-3 antibody (\#9661, 1:3,000), anti-PARP antibody (\#9542, 1:3,000), anti-Apaf-1 (\#8969, 1:2,000) antibody, anti-AIF antibody (\#4642, 1:2,000), anti-Atg12 antibody (\#4180, 1:3,000), anti-Beclin-1 antibody (\#3495, 1:3,000), and anti-LC3A/B antibody (\#12741, 1:200) were purchased from Cell Signaling Technology. Anti-Myc antibody (9E10, sc-40, 1:3,000) and anti-lamin A antibody (4A58, sc-71481, 1:2,000) were purchased from Santa Cruz. The anti-FLAG antibody (M2, F1804, 1:3,000), anti-tubulin antibody (DM1A, T9016, 1:3,000) and anti- $\beta$-actin (A2066, 1:3,000) antibody were purchased from Sigma. The anti-GFP antibody (ab6556, 1:3,000) was from Abcam.

Nuclear fractionation. Cultured cells were washed with PBS, homogenized in buffer A [10 mM HEPES (pH 7.4), $1.5 \mathrm{mM}$ $\mathrm{MgCl}_{2}, 10 \mathrm{mM} \mathrm{KCl}, 0.5 \mathrm{mM}$ DTT, $0.1 \%$ NP-40], and centrifuged at $600 \mathrm{x}$ g for $10 \mathrm{~min}$ at $4^{\circ} \mathrm{C}$. The nuclear pellet was washed with buffer A, resuspended in buffer $\mathrm{C}$ [10 mM HEPES (pH 7.4), $1.5 \mathrm{mM} \mathrm{MgCl}_{2}, 0.5 \mathrm{mM}$ DTT, $20 \%$ glycerol, $0.5 \mathrm{mM}$ PMSF, $0.2 \mathrm{mM}$ EDTA, and $420 \mathrm{mM} \mathrm{NaCl}$ ], and centrifuged for $30 \mathrm{~min}$ at $15,000 \mathrm{rpm}$, before the supernatant was isolated.

Immunoblotting and immunoprecipitation. Proteins were extracted using lysis buffer consisting of $20 \mathrm{mM}$ Tris (pH 7.5),
$150 \mathrm{mM} \mathrm{NaCl}, 0.1 \mathrm{mM}$ EDTA, $0.2 \%$ Triton $\mathrm{X}-100$ and a protease inhibitor cocktail (Roche). Then, $20 \mathrm{mg}$ of cell extract was used for immunoblotting. For immunoprecipitation, $1 \mathrm{mg}$ of protein was incubated with a corresponding antibody conjugated to A or $\mathrm{G}$ beads (Upstate) overnight at $4^{\circ} \mathrm{C}$. Beads were washed three times with washing buffer containing $20 \mathrm{mM}$ Tris (pH 7.5), $150 \mathrm{mM} \mathrm{NaCl}$ and $0.1 \mathrm{mM}$ EDTA. Following SDS-PAGE, membranes were immunoblotted using the corresponding primary antibody overnight at $4^{\circ} \mathrm{C}$. HRP-conjugated secondary antibodies were incubated with the membranes for $1 \mathrm{~h}$ at room temperature. Visualization was performed using ECL Plus (Intron) and LAS-4000 (GE Healthcare).

Immunocytochemistry. HeLa cells were seeded onto glass coverslips in 24-well plates and transfected with GFP-Hsp70 WT and K77R. After treatment of $0.3 \mathrm{mM} \mathrm{H}_{2} \mathrm{O}_{2}$ for $24 \mathrm{~h}$, cells were fixed in 4\% PFA for $20 \mathrm{~min}$ and permeabilized in $0.3 \%$ Triton X-100 in PBS for $5 \mathrm{~min}$ at room temperature. Then, cells were incubated with LC3A/B antibody (Cell Signaling Technology, \#12741, 1:200) and visualized with Alexa 546-conjugated IgG (Molecular Probes). Nuclear staining was performed using Hoechst 33342 (Molecular Probes). The immunofluorescence was visualized using an Axiovert M200 microscope (Carl Zeiss).

Cell viability assay. Cell viability was calculated by measuring the amount of lactate dehydrogenase (LDH) released from the cells into the medium. Conditioned media from cultured cells were collected, and LDH activity was determined with an LDH assay kit (DoGen). Total cellular LDH activity was measured by solubilizing the cells with $0.2 \%$ Triton X-100.

Statistical analysis. Results are expressed as the means \pm SD P-values were calculated by applying the two-tailed Student's t-test. A difference was considered statistically significant at $\mathrm{P}<0.05$.

\section{Results}

Hsp70 acetylation protects cancer cells from doxorubicininduced cell death. Hsp70 acetylation at residue $\mathrm{K} 77$ has been previously reported to protect cells from stress. To confirm the protective effect of Hsp70 acetylation, we treated Hsp70 wild-type (WT) and K77R mutant-expressing SH-SY5Y cells with doxorubicin and analyzed cell viability. Consistent with a previous report, overexpression of wild-type Hsp70 protected cells against doxorubicin-induced cell death, but this protective effect was eliminated by the presence of the K77R mutation (Fig 1A). The acetylation of Hsp70 at K77 is mediated by acetyltransferase ARD1 (19). To further validate the relevance of ARD1 in the protective function of Hsp70, ARD1 WT and a dominant-negative mutant (DN) that does not harbor acetyltransferase activity was co-expressed with Hsp70 constructs in SH-SY5Y cells, and cell viability was assessed after doxorubicin treatment (21). As expected, co-expression of the dominant-negative mutant ARD1 abolished the protective effect of Hsp70 WT (Fig. 1B), indicating that ARD1-mediated Hsp70 acetylation protects cancer cells from doxorubicininduced cell death. 
A

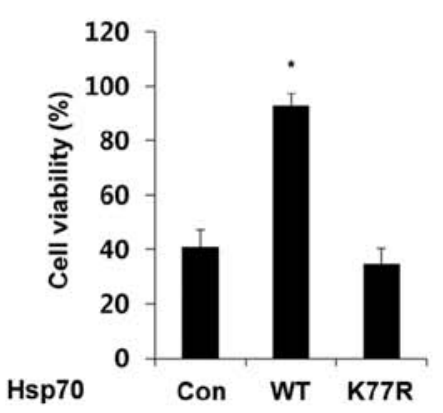

Hsp70

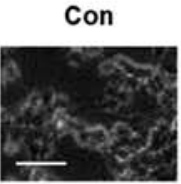

WT

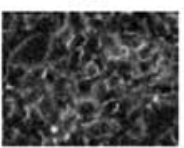

K77R

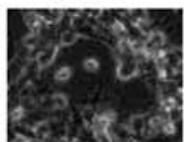

B

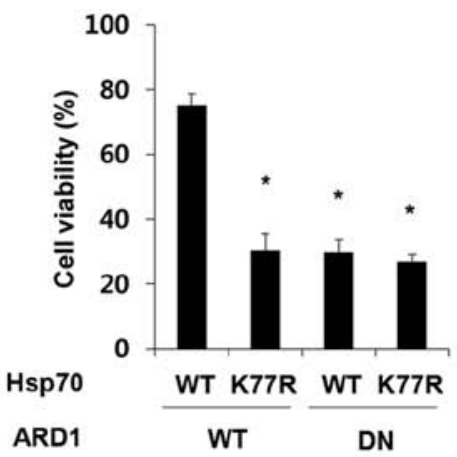

Hsp70

ARD1

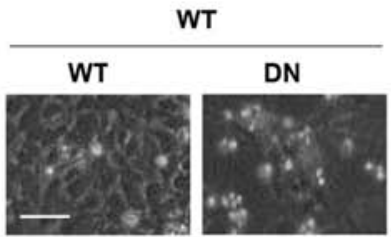

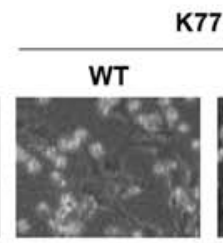

K77R DN

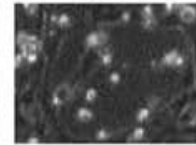

Figure 1. Hsp70 acetylation inhibits doxorubicin-induced cancer cell death. (A) Hsp70 acetylation promotes cell survival during doxorubicin challenge. SH-SY5Y cells expressing GFP-Hsp70 WT and K77R were treated with $1 \mu \mathrm{g} / \mathrm{ml}$ doxorubicin for $24 \mathrm{~h}$ and cell survival was assessed. Error bars indicate $\mathrm{SD}(\mathrm{n}=3)$. ${ }^{*} \mathrm{P}<0.05$ versus control (t-test). Scale bar, $50 \mu \mathrm{m}$. (B) ARD1 promotes cell survival via Hsp70 acetylation following doxorubicin treatment. FLAG-ARD1 WT and dominant negative (DN) forms were co-expressed with GFP-Hsp70 in SH-SY5Y cells. Cell viability was measured after 24-h treatment of $1 \mu \mathrm{g} / \mathrm{ml}$ doxorubicin. Error bars indicate $\mathrm{SD}(\mathrm{n}=3)$. ${ }^{*} \mathrm{P}<0.05$ versus Hsp70 WT/ARD1 WT (t-test). Scale bar, $50 \mu \mathrm{m}$.

A

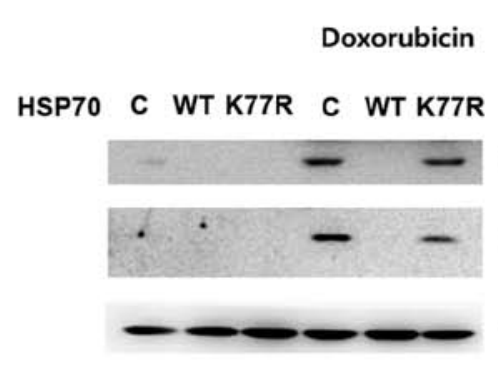

B

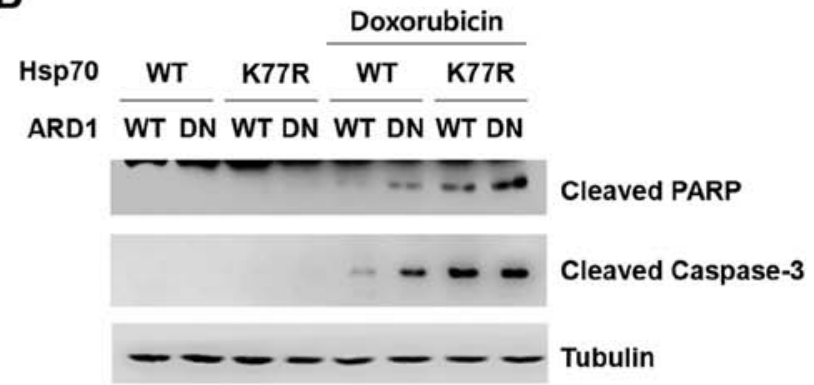

C

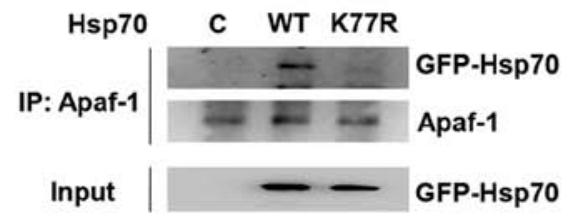

Figure 2. Hsp70 acetylation prevents caspase-dependent apoptotic cell death. (A) Hsp70 acetylation inhibits stress-induced cleavage of caspase-3 and PARP. After treatment of $1 \mu \mathrm{g} / \mathrm{ml}$ doxorubicin for $24 \mathrm{~h}$, caspase-3 and PARP cleavage was assessed by western blotting. (B) ARD1 inhibits caspase-3 and PARP cleavage via Hsp70 acetylation. SH-SY5Y cells co-expressing FLAG-ARD1 and Hsp70 were treated with $1 \mu \mathrm{g} / \mathrm{ml}$ of doxorubicin for $24 \mathrm{~h}$, and caspase-3 and PARP cleavage was analyzed. (C) Hsp70 acetylation is required for Apaf-1 binding. Apaf-1 was precipitated from HEK293T cells overexpressing GFP-Hsp70 WT and K77R, and the co-precipitated GFP-Hsp70 was quantified.

Hsp70 acetylation inhibits caspase-dependent apoptosis. To further investigate the underlying mechanisms of Hsp70 acetylation-mediated cellular protection, we first analyzed caspase-dependent apoptosis. Doxorubicin treatment induced cleavage of caspase-3 and poly(ADP-ribose) polymerase (PARP), which are hallmarks of apoptosis (Fig. 2A). Consistent with the doxorubicin-induced cell death shown in Fig. 1, Hsp70 WT overexpression prevented doxorubicin-induced cleavage of caspase-3 and PARP, whereas the presence of the K77R mutation abolished the protective effect. Moreover, co-expression of the dominant-negative mutant ARD1 diminished Hsp70 acetylation-mediated inhibition of PARP and caspase cleavage (Fig. 2B).

Since Hsp70 is reported to inhibit apoptosome formation via binding with Apaf-1, a key molecule of caspase-dependent apoptosis, we next investigated whether acetylation at K77 can 
A

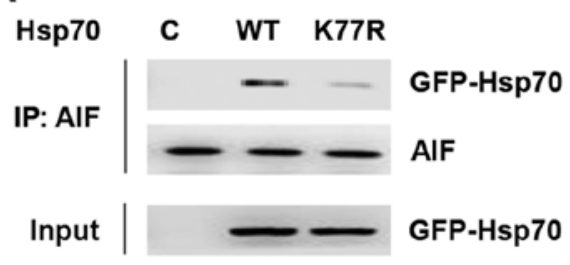

B

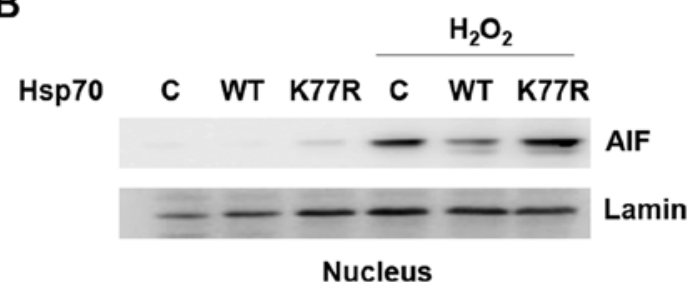

Figure 3. Hsp70 acetylation inhibits AIF-dependent apoptosis. (A) Hsp70 acetylation is required for AIF binding. AIF was precipitated from HEK293T cells, and co-precipitation of GFP-Hsp70 WT or K77R was assessed by western blotting. (B) Hsp70 acetylation inhibits nuclear translocation of AIF. SH-SY5Y cells expressing GFP-Hsp70 WT or K77R were treated in the presence or absence of $0.3 \mathrm{mM} \mathrm{H}_{2} \mathrm{O}_{2}$, and nuclear proteins were fractionated and assessed by western blotting.

modulate Apaf-1 binding. Hsp70 WT or the K77R mutant was overexpressed in HEK293T cells and their affinity to Apaf-1 was assessed by co-immunoprecipitation. Consistent with previous reports, Hsp70 wild-type was co-immunoprecipitated with Apaf-1 in cell extracts. Interestingly, however, mutation at K77 abrogated its binding to Apaf-1, implying that Hsp70 acetylation contributes to Hsp70/Apaf-1 association, subsequently leading to inhibition of functional apoptosome assembly and caspase activation (Fig. 2C). These results suggest that Hsp70 acetylation enables the association of Apaf-1 with Hsp70 and prevents apoptosis.

Hsp70 acetylation prevents caspase-independent apoptosis. Another cell death pathway in which Hsp70 is involved is AIF-dependent apoptosis. Hsp70 inhibits caspase-independent cell death by sequestering AIF and blocking its induction of apoptosis. To examine its relevance to caspase-independent apoptosis, we analyzed AIF binding to Hsp70 WT and the K77R mutant by co-immunoprecipitation (Fig. 3A). Interestingly, compared to Hsp70 WT, AIF binding to the K77R mutant was significantly reduced, implying that Hsp70 acetylation can interfere with AIF-mediated caspase-independent apoptosis.

We next verified whether this change in binding affinity was indeed relevant for AIF function. Nuclear translocation of AIF is a key final step in the AIF pathway (14). To validate the difference in binding affinity and whether it influences AIF nuclear translocation, Hsp70-expressing cells were treated with hydrogen peroxide and nuclear translocation of AIF was greatly increased (Fig. 3B). Moreover, Hsp70 WT reduced $\mathrm{H}_{2} \mathrm{O}_{2}$-induced nuclear translocation of AIF but the K77R mutant could not. These results demonstrate that Hsp70 K77 acetylation suppresses apoptosis by preventing AIF from nuclear translocation, consequently inhibiting caspaseindependent cell death. Collectively, these findings indicate that Hsp70 acetylation protects cancer cells against apoptosis through inhibition of both caspase-dependent and -independent apoptotic pathways.

Hsp70 acetylation attenuates autophagic cell death. Autophagic cell death is another type of programmed cell death that is controlled by Hsp70. Recent studies of stress-induced Hsp70 acetylation and its modulatory role in autophagosome formation led us to hypothesize that Hsp70 acetylation at K77 can also regulate autophagy (22). To test this, we treated $\mathrm{H}_{2} \mathrm{O}_{2}$ to Hsp70 WT and K77R mutant-expressing cells and assessed autophagy-related proteins. Autophagy-related genes (Atg) are universal markers for autophagic induction (23). During stepwise autophagy induction, Beclin-1 (the mammalian orthologue of yeast Atg6)-mediated core complex formation and Atg12-Atg5 conjugation are key processes, in nucleation and elongation of autophagosome formation, respectively $(24,25)$. Consistent with previous reports, Hsp70 WT overexpression decreased $\mathrm{H}_{2} \mathrm{O}_{2}$-induced Beclin-1 and Atg12-Atg5 conjugation, suggesting that Hsp70 plays a regulatory role in the modulation of autophagy (Fig. 4A). However, the Hsp70 K77R mutant could not prevent autophagy as demonstrated by an increase in Beclin-1 and Atg12-Atg5 conjugation. This observation implies that Hsp70 acetylation contributes to the attenuation of autophagic cell death.

We also analyzed microtubule-associated protein light chain 3 (LC3) to monitor autophagic induction. Upon autophagy, the unconjugated cytosolic form of LC3-I is converted to the phosphatidylethanolamine-conjugated form of LC3-II that forms the autophagosomal membrane $(26,27)$. Therefore, the transition of LC3 from a diffusive cytoplasm pattern to the punctated membrane pattern is a hallmark of autophagy induction, indicating the formation of autophagic vacuoles (27). When compared to WT, the Hsp70 mutant caused an increase in LC3 expression and perinuclear autophagic vacuole formation (Fig. 4B). These results suggest that Hsp70 acetylation may play a role in the prevention of autophagic cell death.

\section{Discussion}

In response to stress, Hsp70 acetylation on K77 residue facilitated Hsp70 interaction with Apaf-1 and AIF, and inhibited Apaf-1 and AIF-dependent apoptosis. Moreover, acetylation of Hsp70 attenuated autophagy, observed via Atg12-Atg5 complex formation, Beclin-1 expression and perinuclear LC3 puncta formation, resulting in the inhibition of autophagic cell death. Taken together, our results suggest that Hsp70 acetylation inhibits cell death by at least three different mechanisms: i) attenuation of caspase-dependent pathways by interacting with Apaf-1 and blocking apoptosome formation, ii) inhibition of caspase-independent pathways by interacting with AIF and preventing its nuclear translocation, and iii) attenuation of autophagic cell death (Fig. 5).

Previously, Hsp70 acetylation induced by cellular stress was reported to increase its protein refolding chaperone activity. This is mediated by increased association of Hsp70 with co-chaperones assisting protein refolding such as Hsp90 
A

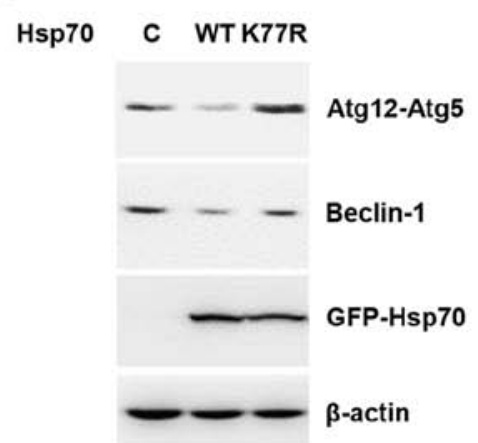

B

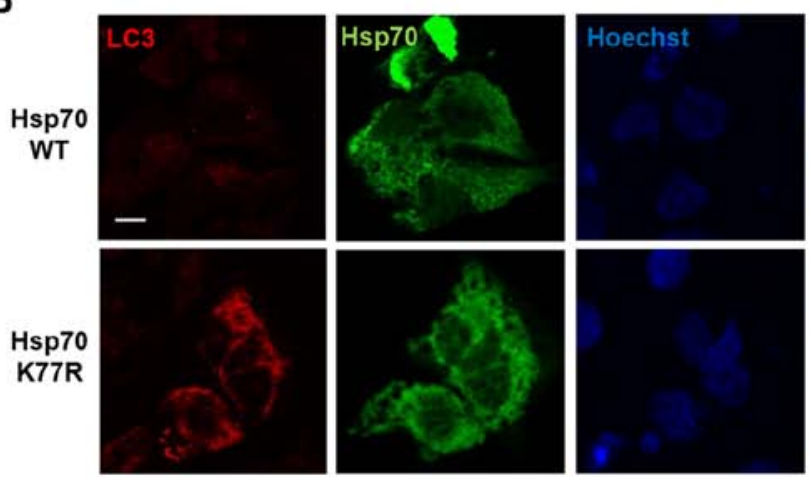

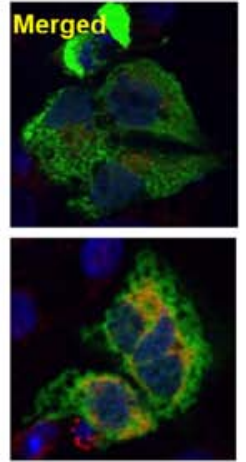

Figure 4. Hsp70 acetylation attenuates autophagic cell death. (A) Hsp70 acetylation reduces autophagy induction. Hsp70 WT and K77R-expressing SH-SY5Y cells were treated with $0.3 \mathrm{mM} \mathrm{H}_{2} \mathrm{O}_{2}$ for $24 \mathrm{~h}$, and cell lysates were subjected to western blotting. (B) Hsp70 acetylation attenuates stress-induced autophagosome formation. Hsp70 WT and K77R-expressing HeLa cells were treated with $0.3 \mathrm{mM} \mathrm{H}_{2} \mathrm{O}_{2}$ for $24 \mathrm{~h}$, and LC3 patterns were assessed by immunofluorescence staining. Nuclei were labeled with Hoechst (blue). Scale bar, $10 \mu \mathrm{m}$.

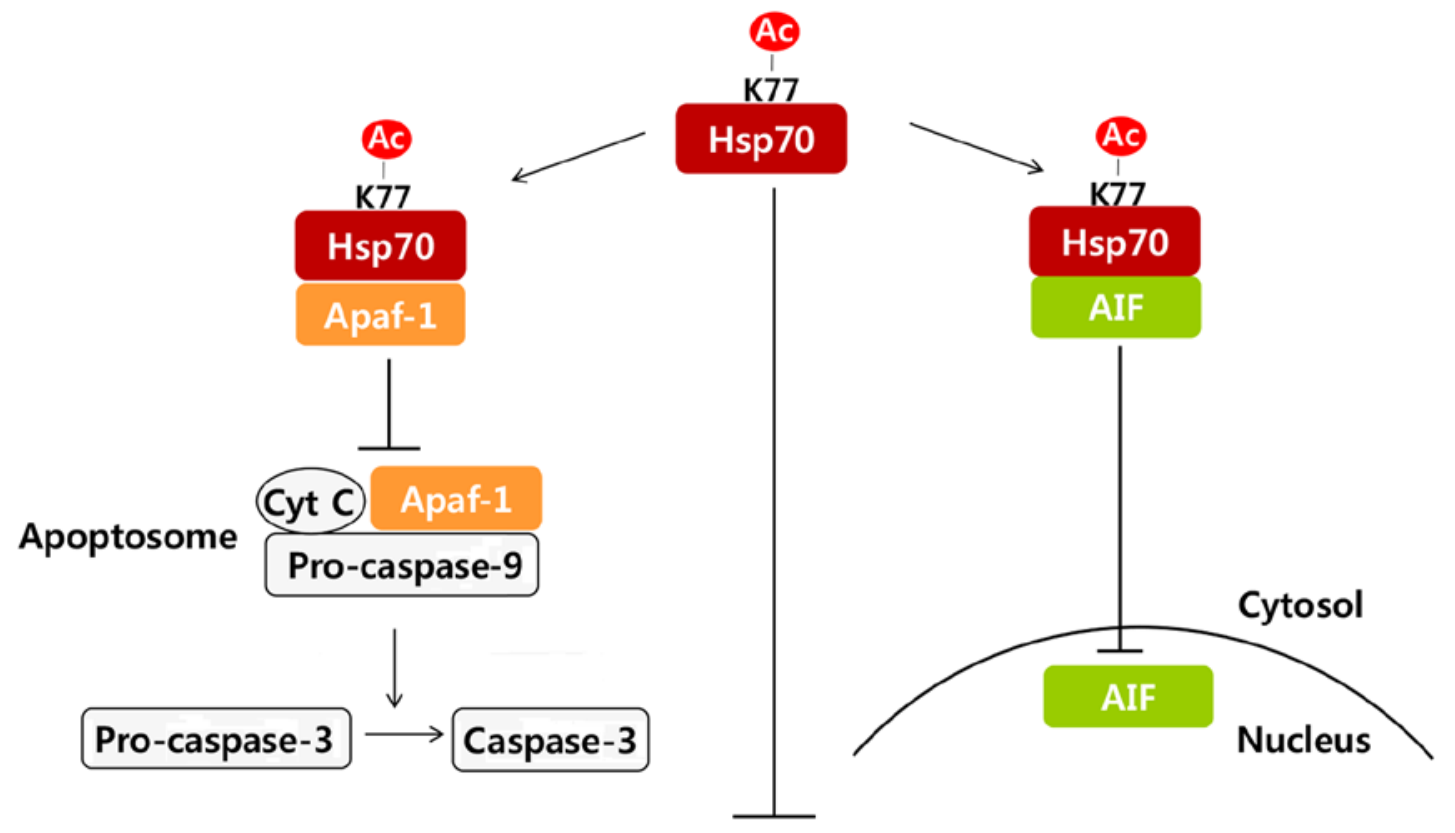

\section{Caspase-dependent cell death}

Autophagy

\section{Caspase-independent cell death}

Figure 5. Model of prevention of cell death regulated by Hsp70 acetylation at K77. During stress, Hsp70 acetylation inhibits cell death by facilitating Apaf-1 and AIF binding to prevent caspase-dependent and -independent apoptosis and attenuating autophagic cell death.

and Hop (19). In addition to co-chaperone binding, in this study, Hsp70 acetylation facilitated binding of pro-apoptotic proteins as well, implying a broad impact for Hsp70 acetylation on its overall functionality.

Autophagy, together with HSP systems, represents a major protein quality control system. To cope with stress-induced cell damage, Hsp70 maintains protein homeostasis primarily by facilitating protein refolding and prevent aggregation, while autophagy results in protein and whole-organelle degradation. However, the role of autophagy in cell death and survival has long been controversial $(3,28)$. It has been accepted as a cell survival mechanism in response to cellular stresses like starvation. However, recent molecular approaches have provided evidence that autophagy contributes to programmed cell death $(29,30)$. Hsp70 has been suggested to play a crucial role in autophagy regulation, although the underlying mechanisms need further investigation $(18,22)$. This study elucidates Hsp70 acetylation as a new regulatory mechanism in autophagic induction and also adds evidence for the contribution of autophagy to programmed cell death. Furthermore, it also suggests the possible linkage between HSP and the autophagy system mediated by Hsp70 acetylation, although the precise causality in physiological signals and underlying mechanisms requires further investigation.

How Hsp70 acetylation at K77 can affect its target protein affinity is another issue that needs to be addressed. The nucleotide binding domain (NBD) of Hsp70 that contains $\mathrm{K} 77$ is required for Hsp70/Apaf-1 interaction, whereas the 
Hsp70/AIF interaction appears to be independent of NBD (13). Previously, we suggested that K77 acetylation in NBD may induce allosteric conformational changes in other domains of Hsp70, resulting in overall changes to target protein binding. The significant location of acetylation site K77 at interdomain contacts increases the interesting possibility that acetylation may modulate the Hsp70 conformational changes important for its protein domain interactions and overall activity. Although detailed studies are needed to elucidate the exact mechanisms involved, our results provide insight into the acetylation-mediated allosteric regulation of Hsp70.

In conclusion, we have described cancer cell survival mechanisms mediated by Hsp70 acetylation under stress. The findings offer rationale for the development of an Hsp70 inhibitor that minimizes disturbance of the normal cellular function of Hsp70. Regulation of Hsp70 K77 acetylation might be helpful in treating various diseases that involve Hsp70, including cancer, inflammatory diseases and neurodegenerative diseases.

\section{Acknowledgements}

This study was supported by the Global Research Laboratory Program (2011-0021874), Brain Korea 21 Program, the Global Core Research Center (GCRC) Program (20110030001), Bio \& Medical Technology Development Program (2015M3A9E6028949), NRF grant (2015R1C1A2A01054446) through the National Research Foundation of Korea (NRF) funded by the Ministry of Science, ICT and Future Planning (MSIP), and Basic Science Research Program (2013R1A1A2058956, 2016R1D1A1B03935560) through the NRF funded by the Ministry of Education.

\section{References}

1. Wang $M$ and Kaufman RJ: The impact of the endoplasmic reticulum protein-folding environment on cancer development. Nat Rev Cancer 14: 581-597, 2014

2. Jezierska-Drutel A, Rosenzweig SA and Neumann CA: Role of oxidative stress and the microenvironment in breast cancer development and progression. Adv Cancer Res 119: 107-125, 2013.

3. Xu Y, Xia X and Pan H: Active autophagy in the tumor microenvironment: A novel mechanism for cancer metastasis. Oncol Lett 5: 411-416, 2013.

4. Vaughn AE and Deshmukh M: Glucose metabolism inhibits apoptosis in neurons and cancer cells by redox inactivation of cytochrome $c$. Nat Cell Biol 10: 1477-1483, 2008.

5. Liu Y, Borchert GL, Donald SP, Surazynski A, Hu CA, Weydert CJ, Oberley LW and Phang JM: MnSOD inhibits proline oxidase-induced apoptosis in colorectal cancer cells. Carcinogenesis 26: 1335-1342, 2005.

6. Fodale V, Pierobon M, Liotta L and Petricoin E: Mechanism of cell adaptation: When and how do cancer cells develop chemoresistance? Cancer J 17: 89-95, 2011.

7. Murphy ME: The HSP70 family and cancer. Carcinogenesis 34 : 1181-1188, 2013

8. Goldberg AL: Protein degradation and protection against misfolded or damaged proteins. Nature 426: 895-899, 2003.

9. Hartl FU, Bracher A and Hayer-Hartl M: Molecular chaperones in protein folding and proteostasis. Nature 475: 324-332, 2011.

10. Beere HM, Wolf BB, Cain K, Mosser DD, Mahboubi A, Kuwana T, Tailor P, Morimoto RI, Cohen GM and Green DR: Heat-shock protein 70 inhibits apoptosis by preventing recruitment of procaspase-9 to the Apaf-1 apoptosome. Nat Cell Biol 2: $469-475,2000$
11. Saleh A, Srinivasula SM, Balkir L, Robbins PD and Alnemri ES: Negative regulation of the Apaf-1 apoptosome by Hsp70. Nat Cell Biol 2: 476-483, 2000.

12. Ravagnan L, Gurbuxani S, Susin SA, Maisse C, Daugas E, Zamzami N, Mak T, Jäättelä M, Penninger JM, Garrido C, et al: Heat-shock protein 70 antagonizes apoptosis-inducing factor. Nat Cell Biol 3: 839-843, 2001.

13. Candé C, Cohen I, Daugas E, Ravagnan L, Larochette N, Zamzami N and Kroemer G: Apoptosis-inducing factor (AIF): A novel caspase-independent death effector released from mitochondria. Biochimie 84: 215-222, 2002.

14. Daugas E, Susin SA, Zamzami N, Ferri KF, Irinopoulou T, Larochette N, Prévost MC, Leber B, Andrews D, Penninger J, et al: Mitochondrio-nuclear translocation of AIF in apoptosis and necrosis. FASEB J 14: 729-739, 2000.

15. Liu Y and Levine B: Autosis and autophagic cell death: The dark side of autophagy. Cell Death Differ 22: 367-376, 2015.

16. Tsujimoto Y and Shimizu S: Another way to die: Autophagic programmed cell death. Cell Death Differ 12 (Suppl 2): 1528-1534, 2005.

17. Glick D, Barth S and Macleod KF: Autophagy: Cellular and molecular mechanisms. J Pathol 221: 3-12, 2010.

18. Dokladny K, Zuhl MN, Mandell M, Bhattacharya D, Schneider S, Deretic V and Moseley PL: Regulatory coordination between two major intracellular homeostatic systems: Heat shock response and autophagy. J Biol Chem 288: 14959-14972, 2013.

19. Seo JH, Park JH, Lee EJ, Vo TT, Choi H, Kim JY, Jang JK, Wee HJ, Lee HS, Jang SH, et al: ARD1-mediated Hsp70 acetylation balances stress-induced protein refolding and degradation. Nat Commun 7: 12882, 2016

20. Cha JH, Wee HJ, Seo JH, Ahn BJ, Park JH, Yang JM, Lee SW, Lee OH, Lee HJ, Gelman IH, et al: Prompt meningeal reconstruction mediated by oxygen-sensitive AKAP12 scaffolding protein after central nervous system injury. Nat Commun 5: 4952, 2014.

21. Seo JH, Park JH, Lee EJ, Vo TT, Choi H, Jang JK, Wee HJ, Ahn BJ, Cha JH, Shin MW, et al: Autoacetylation regulates differentially the roles of ARD1 variants in tumorigenesis. Int J Oncol 46: 99-106, 2015

22. Yang Y, Fiskus W, Yong B, Atadja P, Takahashi Y, Pandita TK, Wang HG and Bhalla KN: Acetylated hsp70 and KAP1-mediated Vps34 SUMOylation is required for autophagosome creation in autophagy. Proc Natl Acad Sci USA 110: 6841-6846, 2013.

23. Lamb CA, Yoshimori T and Tooze SA: The autophagosome: Origins unknown, biogenesis complex. Nat Rev Mol Cell Biol 14: 759-774, 2013.

24. Kang R, Zeh HJ, Lotze MT and Tang D: The Beclin 1 network regulates autophagy and apoptosis. Cell Death Differ 18: 571-580, 2011.

25. Hanada T, Noda NN, Satomi Y, Ichimura Y, Fujioka Y, Takao T, Inagaki $\mathrm{F}$ and Ohsumi $\mathrm{Y}$ : The Atg12-Atg5 conjugate has a novel E3-like activity for protein lipidation in autophagy. J Biol Chem 282: 37298-37302, 2007.

26. Tanida I, Ueno T and Kominami E: LC3 conjugation system in mammalian autophagy. Int J Biochem Cell Biol 36: 2503-2518, 2004.

27. Lamoureux F, Thomas C, Crafter C, Kumano M, Zhang F, Davies BR, Gleave ME and Zoubeidi A: Blocked autophagy using lysosomotropic agents sensitizes resistant prostate tumor cells to the novel Akt inhibitor AZD5363. Clin Cancer Res 19: 833-844, 2013.

28. Yonekawa T and Thorburn A: Autophagy and cell death. Essays Biochem 55: 105-117, 2013

29. Shimizu S, Kanaseki T, Mizushima N, Mizuta T, ArakawaKobayashi S, Thompson CB and Tsujimoto Y: Role of Bcl-2 family proteins in a non-apoptotic programmed cell death dependent on autophagy genes. Nat Cell Biol 6: 1221-1228, 2004.

30. Yu L, Alva A, Su H, Dutt P, Freundt E, Welsh S, Baehrecke EH and Lenardo MJ: Regulation of an ATG7-beclin 1 program of autophagic cell death by caspase-8. Science 304: 1500-1502, 2004. 